\title{
Optical conductivity of the type-II Weyl semimetal $\mathrm{WTe}_{2}$ under pressure
}

\author{
M. Krottenmüller, ${ }^{1}$ J. Ebad-Allah,,${ }^{1,2}$ V. Süss, ${ }^{3}$ C. Felser, ${ }^{3}$ and C. A. Kuntscher ${ }^{1, \text { * }}$ \\ ${ }^{1}$ Experimentalphysik II, Institute of Physics, University of Augsburg, 86159 Augsburg, Germany \\ ${ }^{2}$ Department of Physics, Tanta University, 31527 Tanta, Egypt \\ ${ }^{3}$ Max Planck Institute for Chemical Physics of Solids, \\ Nöthnitzer Strasse 40, 01187 Dresden, Germany
}

\begin{abstract}
Tungsten ditelluride $\mathrm{WTe}_{2}$ is a type-II Weyl semimetal with electronic properties highly sensitive to external pressure, as demonstrated by the superconductivity emerging under pressure. Here, we study the optical conductivity of the type-II Weyl semimetal $\mathrm{WTe}_{2}$ under external pressure at room temperature. With increasing pressure, a pronounced spectral weight transfer occurs from the highenergy to the low-energy interband transitions, with drastic changes in the profile of the optical conductivity spectrum indicating a high sensitivity of the electronic band structure to external pressure. The detailed analysis of the pressure-dependent optical conductivity furthermore reveals anomalies at the pressures $\sim 2$ and $\sim 4.5 \mathrm{GPa}$, where an electronic and a structural phase transition, respectively, were reported in the literature.
\end{abstract}

\section{INTRODUCTION}

The layered transition-metal dichalcogenide $\mathrm{WTe}_{2}$ is currently extensively studied [1 3], as it is an inversionsymmetry-breaking type-II Weyl semimetal [4 [6] with an extremely large nonsaturating magnetoresistance (MR) [7-10] and other outstanding properties, such as a nonlinear anomalous Hall effect in few-layer samples, despite being non-magnetic [11, 12], room-temperature ferroelectricity [13], unconventional Nernst effect [14], and pressure-induced superconductivity [15, 16]. An ultrafast symmetry switch has been realized in $\mathrm{WTe}_{2}$ [17, exploiting structural changes induced by teraherz radiation and the accompanying changes in the topological Weyl state. The large MR in $\mathrm{WTe}_{2}$ is usually explained by a perfect compensation of electron and hole carriers [7], which is supported by theoretical calculations [18] as well as ARPES and transport experiments 19 22]. However, the results of Hall effect measurements questioned this scenario [23, 24] and alternative explanations such as strong spin-orbit coupling and forbidden backscatterings due to the spin texture were proposed [25, 26]. Therefore, knowledge of the properties of the Fermi surface of $\mathrm{WTe}_{2}$ is highly desirable. According to angle-dependent transport measurements, the electronic properties of $\mathrm{WTe}_{2}$ are rather three-dimensional, and the temperature dependence of the magnetoresistance follows the temperature dependence of the mass anisotropy and thus the anisotropy of the Fermi surface [27]. Furthermore, nonlinear MR and its temperature behavior were explained by the temperature-induced changes in Fermi surface convexity [28].

Shubnikov-de Haas experiments under pressure [20] not only favor the scenario of two electron and two hole pockets of similar size, but also highlight the drastic pressure dependence of the Fermi surface of $\mathrm{WTe}_{2}$. Accordingly, with increasing pressure, a strong increase of the

\footnotetext{
* christine.kuntscher@physik.uni-augsburg.de
}

size of the Fermi surface is observed, as well as a change in its topology, namely two pockets disappear around $1 \mathrm{GPa}$. Interestingly, two other studies on $\mathrm{WTe}_{2}$ discovered superconductivity under pressure with a maximum $\mathrm{T}_{c} \approx 7 \mathrm{~K}$, although with a discrepancy in the pressure onset of the transition $(10.5,2.5 \mathrm{GPa})[15$, 16] coinciding with the suppression of the large MR effect. It was furthermore found that $\mathrm{WTe}_{2}$ undergoes a pressure-induced structural phase transition from the ambient-pressure orthorhombic $\mathrm{T}_{\mathrm{d}}$ to the monoclinic $\mathrm{T}^{\prime}$ phase at $\sim 8 \mathrm{GPa}$ 29 31]. Thus, it was suggested that the structural phase transition separates the large MR state from the superconducting state [30, 31], in contradiction to the findings in Ref. [29], where both polytypes show superconductivity. Furthermore, electronic band structure calculations under pressure show an anisotropic Fermi surface at high pressure [30], whereas recent angle-dependent measurements of the upper critical field reveal a nearly isotropic superconductivity at $\sim 10 \mathrm{GPa}$ [32], not compatible with the calculated Fermi surface anisotropy.

Optical spectroscopy is a powerful technique for probing the charge dynamics in a material with a high energy resolution. In particular, materials with nontrivial topology show a characteristic frequency dependence of their optical conductivity. In particular, 3D Dirac and type I-Weyl semimetals show a frequency-linear behavior due to interband transitions between the linearly dispersing nontrivial bands [33], like it was detected, e.g., for TaAs 34]. In comparison, the optical conductivity of type-II Weyl semimetals with tilted cones contains two regions with quasi-linear behavior, where the change of slope is a measure for the tilting of the Weyl cones [35]. Linear extrapolation of the higher-energy region gives a finite conductivity value at zero frequency.

The optical conductivity of $\mathrm{WTe}_{2}$ at ambient pressure [36 38] contains two Drude components whose spectral weight and scattering rate showed a markedly different temperature behavior. One Drude term was associated with trivial, semimetallic electron and hole bands, while the other one with Dirac carriers at Weyl points [38]. $\mathrm{WTe}_{2}$ also shows a frequency-linear optical conductivity 
typical for Dirac and Weyl semimetals; however, according to ab initio calculations, in $\mathrm{WTe}_{2}$ the linear behavior of the optical conductivity stems from a sum of many transitions involving trivial bands [37], and besides, the two distinct regions with quasi-linear behavior characteristic for type-II Weyl semimetals are lacking.

On the other hand, to our knowledge, such optical signatures for type-II Weyl semimetals could not be unambiguously proven to exist in any candidate material up to now. Although two linear slopes were found in the optical conductivity of $\mathrm{YbMnBi}_{2}$ via reflectivity measurements, both quasi-linear behaviors extrapolate through the origin [39]. Moreover, it was found that a feature which might be attributed to a van Hove singularity in a simplified Weyl semimetal bandstructure, can more likely be described by certain non-idealities in the present material [40]. Two linear regimes separated by a kink were also observed in the optical conductivity of elemental tellurium under pressure [41]. But by band-resolved DFT calculations it was shown that the origin of these features is not due to a Weyl type-II bandstructure. In a recent publication [42], the authors show the optical conductivity of $\mathrm{TaIrTe}_{4}$ indeed consisting of two linear slopes separated by a kink and interpret these findings in terms of a tilted Weyl dispersion, but other origins of this behaviour could not be excluded.

In order to obtain more detailed information on the electronic properties of the type-II Weyl semimetal $\mathrm{WTe}_{2}$ under pressure, we studied the optical conductivity of $\mathrm{WTe}_{2}$ for pressures up to $8 \mathrm{GPa}$. Upon pressure application, apart from the increasing metallicity due to the increase in the Fermi pockets, we observe a large spectral weight transfer from high to low energies. Furthermore, we find indications for two phase transitions, at $\sim 2$ and $\sim 4.5 \mathrm{GPa}$, presumably of electronic and structural type, respectively. Our findings confirm the strong influence of pressure on the bandstructure and thus the peculiar electronic properties of $\mathrm{WTe}_{2}$.

\section{SAMPLE PREPARATION AND EXPERIMENTAL DETAILS}

Single crystals were grown by chemical vapor transport of polycrystalline $\mathrm{WTe}_{2}$ with $\mathrm{TeCl}_{4}$ (Aldrich, 99\%) as a transport additive, the evacuated silica ampoule heated in a two-zone-furnace in a temperature gradient from $900^{\circ} \mathrm{C}$ (T2) to $800^{\circ} \mathrm{C}$ (T1) for several days. After reaction, the ampoule was removed from the furnace and quenched in water. The $0.5-1 \mathrm{~mm}$ size plate-like crystals were characterized by powder x-ray diffraction (XRD). At ambient pressure, $\mathrm{WTe}_{2}$ crystallizes in the noncentrosymmetric, orthorhombic $\mathrm{T}_{\mathrm{d}}$ phase with distorted $\mathrm{Te}$ octahedra and zigzag chains of $\mathrm{W}$ atoms along the $a$ axis [43, 44], in contrast to the monoclinic 1T' structure and the undistorted trigonal prismatic $2 \mathrm{H}$ structure.

Pressure-dependent infrared reflectivity measurements were carried out at room temperature in the frequency range $300-16000 \mathrm{~cm}^{-1}$ using a Bruker Vertex v80 Fourier transform infrared spectrometer coupled to a Bruker Hyperion infrared microscope. For the measurements, freshly cleaved crystals were placed in the hole of $\mathrm{CuBe}$ gaskets inside a screw-driven diamond-anvil cell. Finely ground CsI powder served as quasi-hydrostatic pressure transmitting medium (PTM). The pressure inside the DAC was determined in situ using the ruby luminescence technique [45, 46]. The reflectivity spectra at the samplediamond interface $R_{\mathrm{s}-\mathrm{d}}(\omega)$ were obtained according to $R_{\mathrm{s}-\mathrm{d}}(\omega)=R_{\mathrm{s}-\text { gasket }}(\omega) \times I_{\mathrm{s}}(\omega) / I_{\text {gasket }}(\omega)$, where $I_{\mathrm{s}}(\omega)$ is the intensity of the radiation reflected at the samplediamond interface, $I_{\text {gasket }}$ is the intensity reflected from the $\mathrm{CuBe}$ gasket-diamond interface, and $R_{\mathrm{s}-\text { gasket }}(\omega)$ is the reflectivity of the gasket material for the diamond interface. The frequency range of the diamond multiphonon absorptions $1800-2700 \mathrm{~cm}^{-1}$ was interpolated based on a Drude-Lorentz fitting of the reflectivity data.

The $R_{\mathrm{s}-\mathrm{d}}(\omega)$ spectra were treated via Kramers Kronig (KK) analysis taking into account the sample-diamond interface [47] in order to obtain the complex optical conductivity $\hat{\sigma}(\omega)=\sigma_{1}(\omega)+\mathrm{i} \sigma_{2}(\omega)$ and the complex dielectric function $\hat{\epsilon}(\omega)=\epsilon_{1}(\omega)+\mathrm{i} \epsilon_{2}(\omega)$. To this end, the low-frequency extrapolations of the reflectivity data were based on Drude-Lorentz fitting, whereas for the high energy extrapolation ambient-pressure x-ray atomic scattering functions were utilized [48], adjusted for the sample-diamond interface.

\section{RESULTS}

Pressure-dependent reflectivity $R_{\mathrm{s}-\mathrm{d}}(\omega)$ spectra of $\mathrm{WTe}_{2}$ are depicted in Fig. 1(a). At the lowest pressure $(1.2 \mathrm{GPa})$, the reflectivity drops quite sharply from $\sim 0.75$ at the lowest measured frequency to $\sim 0.3$ at $1500 \mathrm{~cm}^{-1}$. Above $1500 \mathrm{~cm}^{-1}$, the reflectivity decreases monotonically to the value $\sim 0.18$ at the highest measured frequency $\left(16000 \mathrm{~cm}^{-1}\right)$. The features at intermediate frequencies signal the presence of several interband transitions. The reflectivity drop in the low-energy range of the spectrum corresponds to a plasma edge, which sharpens during cooling down [36]. Since the plasma edge is rather smeared out at room temperature and under pressure, no clear plasmon peak appears in the loss function $\Lambda$, defined as $\Lambda=-\operatorname{Im}(1 / \hat{\epsilon})$ (see inset of Fig. 1(b)). Instead, the loss function increases strongly up to $\sim 1600 \mathrm{~cm}^{-1}$, reaching a plateau, and then increases monotonically in a slower fashion.

With increasing pressure, the overall reflectivity increases, indicating an increasing metallicity as it is expected for semimetallic compounds under pressure. The reflectivity increase is stronger at low frequencies compared to high frequencies. The plasma edge in the reflectivity spectrum shifts to higher energies with increasing pressure. Above $\approx 4.5 \mathrm{GPa}$, a shoulder appears at $\approx 4500 \mathrm{~cm}^{-1}$.

The real part of the optical conductivity $\sigma_{1}$, as plot- 


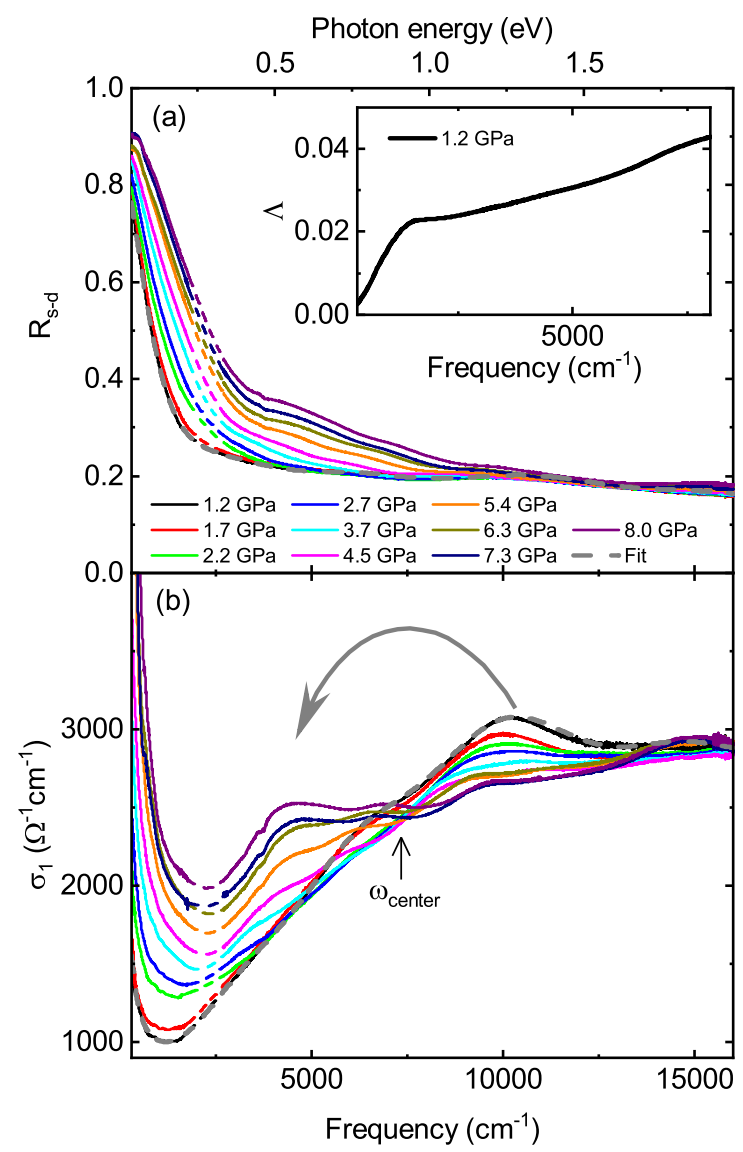

FIG. 1. (a) Reflectivity spectrum $R_{\mathrm{s}-\mathrm{d}}(\omega)$ of $\mathrm{WTe}_{2}$ for all measured pressures, together with the Drude-Lorentz fit for the spectrum at $1.2 \mathrm{GPa}$ (gray dashed line). Inset: Loss function $\Lambda$ at the lowest pressure, showing no clear plasmon peak. (b) Real part of the optical conductivity $\sigma_{1}(\omega)$ of $\mathrm{WTe}_{2}$ for all measured pressures. The gray arrow illustrates the pressure-induced spectral weight transfer from $\omega_{\text {high }}$ to $\omega_{\text {low }}$, as explained in the text. The gray dashed line marks the fit of the optical conductivity spectrum at $1.2 \mathrm{GPa}$.

ted in Fig. 1(b), allows for a more detailed view on the optical properties of $\mathrm{WTe}_{2}$ under pressure. At $1.2 \mathrm{GPa}$, one can see the tail of a small Drude contribution, consistent with the semimetallic nature of $\mathrm{WTe}_{2}$. The value of the optical conductivity at the lowest measured frequency matches well with the dc conductivity of $\sim 1050 \Omega^{-1} \mathrm{~cm}^{-1}$ [49]. Above $1500 \mathrm{~cm}^{-1}$ the optical conductivity increases almost linearly, up to a maximum close to $10000 \mathrm{~cm}^{-1}$. The linear behavior was identified to stem from the sum of many interband transitions involving trivial bands [37], and should not be ascribed to interband transitions close to the Weyl points with a characteristic $\omega$-linear conductivity [33, 35, 50]. Above $10000 \mathrm{~cm}^{-1}$, the optical conductivity slightly decreases first and then remains constant. Overall, the optical conductivity at $1.2 \mathrm{GPa}$ is consistent with the published ambient-pressure data 36 38] 51]. The interband transitions in $\mathrm{WTe}_{2}$ occur between several electronic bands with W-5d and Te-5p character in the vicinity of the Fermi energy $\mathrm{E}_{F}$, including electron and hole bands along the $\Gamma$ - $X$ direction in the Brillouin zone [16, 36]. Furthermore, based on the comparison between the experimental and calculated conductivity spectra, weak electron correlations were inferred [38].

For a quantitative analysis, we carried out a simultaneous fitting of the reflectivity $R_{\mathrm{s}-\mathrm{d}}(\omega)$ and optical conductivity $\sigma_{1}(\omega)$ spectra based on a Drude-Lorentz model for the dielectric function $\hat{\epsilon}(\omega)$, containing one Drude contribution and several Lorentz contributions, according to:

$$
\hat{\epsilon}(\omega)=\epsilon_{\infty}-\frac{\omega_{\mathrm{pl}, \mathrm{D}}^{2}}{\omega^{2}+i \Gamma_{D} \omega}+\sum_{j} \frac{\Omega_{j}^{2}}{\omega_{0, j}^{2}-\omega^{2}-i \Gamma_{\mathrm{j}} \omega},
$$

with the frequency $\omega_{\mathrm{pl}, \mathrm{D}}$ and scattering rate $\Gamma_{D}$ of the Drude contribution, the resonance frequency $\omega_{0, j}$, the oscillator strength $\Omega_{j}$ and the full width at half maximum $\Gamma_{\mathrm{j}}$ of the j-th Lorentz oscillator, and the real part of the dielectric function at high frequency $\epsilon_{\infty}$. Despite two Drude components being considered in optical conductivity spectra of $\mathrm{WTe}_{2} 36$ 38, we included only one Drude term in our analysis, since our measured range starts above $300 \mathrm{~cm}^{-1}$ and the scattering rate of the second Drude term is much smaller [52]. Besides the Drude term, we had to insert a low-energy Lorentzian contribution L1 at $850 \mathrm{~cm}^{-1}$ to adequately describe the low-energy optical conductivity, consistent with recent observations of low-energy excitations in the sister compound $\mathrm{MoTe}_{2}$ [53]. We show the complete fitting with its components for the 1.2 GPa spectrum in Fig. 2(a) (for more information about the fittings see Fig. 5 and 6 in the appendix). According to Ref. [36] the L1 contribution stems from direct transitions between the electronic bands along $\Gamma-X$ associated with the hole and electron pockets. It is difficult to attribute the higher-energy Lorentz contributions to specific electronic transitions, since there are many electronic bands in the energy range $\mathrm{E}_{\mathrm{F}} \pm 1 \mathrm{eV}$ [36].

Importantly, in the region $800-7500 \mathrm{~cm}^{-1}$ the optical conductivity drastically increases with increasing pressure, while it decreases between 7500 and $12500 \mathrm{~cm}^{-1}$ [see Fig. 1(b)]. Above $12500 \mathrm{~cm}^{-1}, \sigma_{1}$ is approximately pressure independent. We also note that at $8 \mathrm{GPa}$ the profile of the optical conductivity $\sigma_{1}$ has markedly changed as compared to ambient pressure, with an almost constant behavior in a broad energy range from 4000 to $16000 \mathrm{~cm}^{-1}$ [see Fig. [(b)]. Since $\sigma_{1}$ is related to the joint density of states JDOS according to $\sigma_{1}(\omega) \propto \operatorname{JDOS}(\omega) / \omega$ assuming frequency-independent transition matrix elements, the observed pressure-induced changes in $\sigma_{1}$ indicate drastic changes in the electronic band structure and cannot be explained by a mere shifting of bands. Overall, one observes a pronounced pressure-induced spectral weight transfer in the optical conductivity spectrum from high to low frequencies around the center frequency $\omega_{\text {center }}=7500 \mathrm{~cm}^{-1}$, approximately forming an isosbestic (equal-absorption) point. Isosbestic points [54, 55] have also been found in the optical conductivity spectrum of stongly correlated materials, like the cuprates 


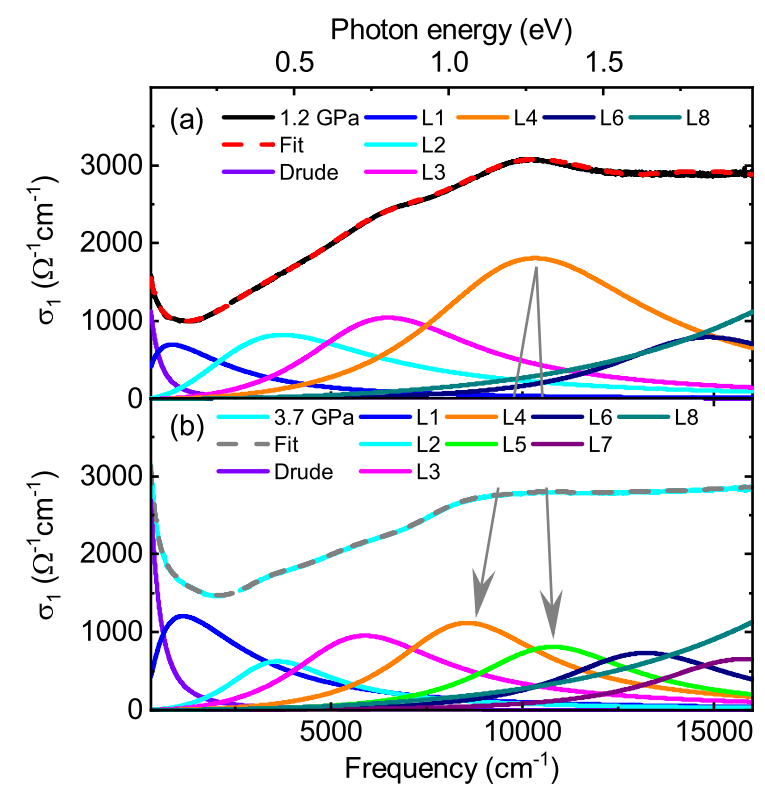

FIG. 2. Optical conductivity spectr together with the fits and contributions for (a) $1.2 \mathrm{GPa}$ and (b) $3.7 \mathrm{GPa}$, illustrating the different models needed to describe the data below and above $2 \mathrm{GPa}$, respectively. The two grey arrows indicate the splitting of the Lorentz contribution L4 into two contributions L4 and L5 above 2 GPa.

$\mathrm{La}_{2-x} \mathrm{Sr}_{x} \mathrm{CuO}_{4}$ [56] and the pyrochlore-type molybdates [57]. Here, they were discussed in terms of a spectral weight transfer between intraband and interband transitions in the context of a Mott-type metal-insulatortransition within the Hubbard model. However, strongly correlated electron physics is not relevant for $\mathrm{WTe}_{2}$.

To quantify the spectral weight transfer related to interband transitions, we subtracted the Drude component from the total optical conductivity for each pressure to obtain the interband contributions to the optical conductivity, $\sigma_{1, \text { inter. Afterwards, we calculated the difference }}$ spectra of the interband transitions $\Delta \sigma_{1, \text { inter }}$ according to $\Delta \sigma_{1, \text { inter }}(\omega, P)=\sigma_{1 \text {,inter }}(\omega, P)-\sigma_{1, \text { inter }}(\omega, 1.2 \mathrm{GPa})$.

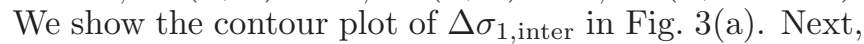
we determined the effective number of electrons $N_{\text {eff }}$ per atom contributing to the interband optical conductivity from the spectral weight analysis, applying the sum rule:

$$
N_{\mathrm{eff}}\left(\omega_{1}, \omega_{\mathrm{u}}\right)=\frac{2 m_{0}}{\pi e^{2} N} \int_{\omega_{1}}^{\omega_{\mathrm{u}}} \sigma_{1, \text { inter }}(\omega) d \omega
$$

where $\omega_{1}$ and $\omega_{\mathrm{u}}$ are the lower and upper limits of the frequency range, respectively, $m_{0}$ is the free electron rest mass, and $N=12$ is the number of atoms per unit cell [58]. We chose the two frequency intervals $\Delta_{\text {low }}$ (800$\left.7500 \mathrm{~cm}^{-1}\right)$ and $\Delta_{\text {high }}\left(7500-12500 \mathrm{~cm}^{-1}\right)$ for our calculations, since the low-frequency interval corresponds to the area around the contribution L2 at $\sim 4500 \mathrm{~cm}^{-1}$ and below, and the high-frequency interval mainly covers the range of the higher energy excitations L4/L5 at $\sim 10000 \mathrm{~cm}^{-1}$. The pressure dependence of the ef-
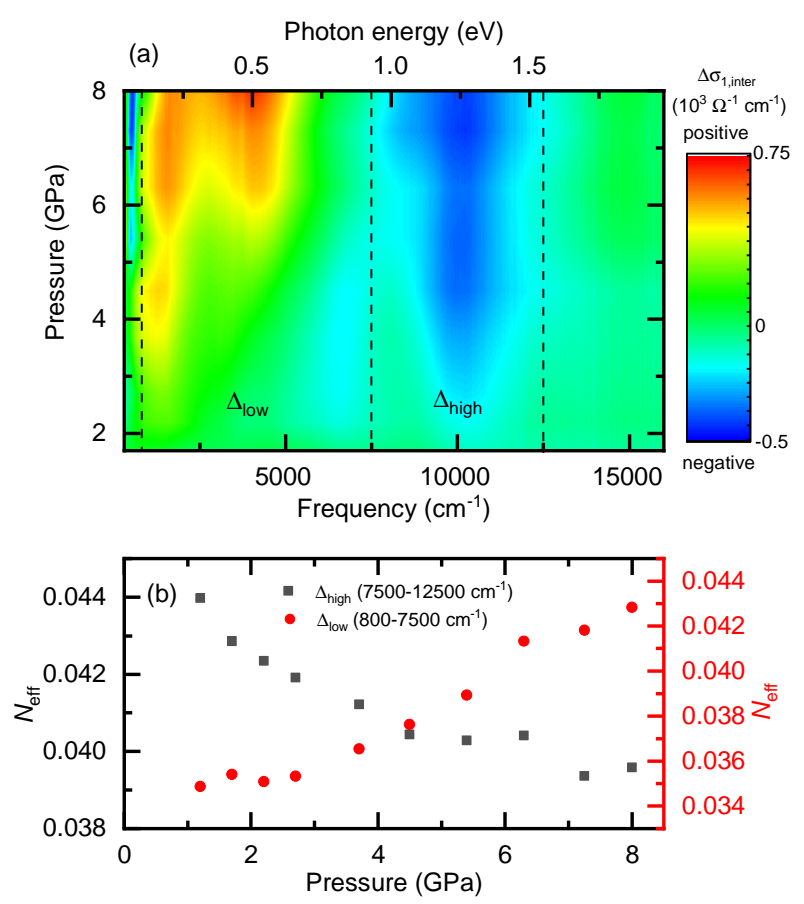

FIG. 3. (a) Contour plot of the difference spectra $\Delta \sigma_{1 \text {,inter }}$ as defined in the text. The vertical dashed lines indicate the frequency intervals $\Delta_{\text {low }}\left(800-7500 \mathrm{~cm}^{-1}\right)$ and $\Delta_{\text {high }}(7500-$ $\left.12500 \mathrm{~cm}^{-1}\right)$. (b) Effective number of electrons in the frequency intervals $\Delta_{\text {low }}$ and $\Delta_{\text {high }}$ as a function of pressure, showing the spectral weight transfer from high to low energies with increasing pressure. The vertical grey shaded areas mark the critical pressure ranges of the phase transitions as explained in the text.

fective number of electrons, corresponding to these two frequency ranges, is plotted in Fig. 3(b). While $N_{\text {eff }}$ for $\Delta_{\text {low }}$ is increasing with increasing pressure, $N_{\text {eff }}$ for $\Delta_{\text {high }}$ is decreasing by approximately the same amount. Since $\sigma_{1}\left(\omega_{\text {center }}\right)$, i.e., in the region between $\Delta_{\text {low }}$ and $\Delta_{\text {high }}$, stays constant throughout all pressures, this behavior rules out a simple shifting of bands. Further bandstructure-related and band-selective optical conductivity calculations are needed in order to identify the pressure-induced changes in the electronic bandstructure.

In addition to the pronounced spectral weight transfer between the interband transitions, we observe a peculiar pressure behavior of the plasma frequency of the Drude component $\omega_{\mathrm{pl}, \mathrm{D}}$, as extracted from the fittings, which is plotted in Fig. 4(a). The plasma frequency is linked to the carrier density $n$ according to $\omega_{\mathrm{pl}, \mathrm{D}}^{2}=4 \pi n e^{2} / \mathrm{m}^{*}$, with the electron charge $e$ and the effective mass $m^{*}$. At $1.2 \mathrm{GPa}, \omega_{\mathrm{pl}, \mathrm{D}}$ amounts to $6396 \pm 205 \mathrm{~cm}^{-1}$, which matches well with literature data at ambient conditions [37. The pressure dependence of $\omega_{\mathrm{pl}, \mathrm{D}}$ shows two anomalies: Above $2 \mathrm{GPa}$, the $\omega_{\mathrm{pl}, \mathrm{D}}$ increases significantly, consistent with the increasing metallicity of $\mathrm{WTe}_{2}$ under pressure [16, 20]. Besides the onset of the pressureinduced increase at $\sim 2 \mathrm{GPa}$, there seems to be a sec- 


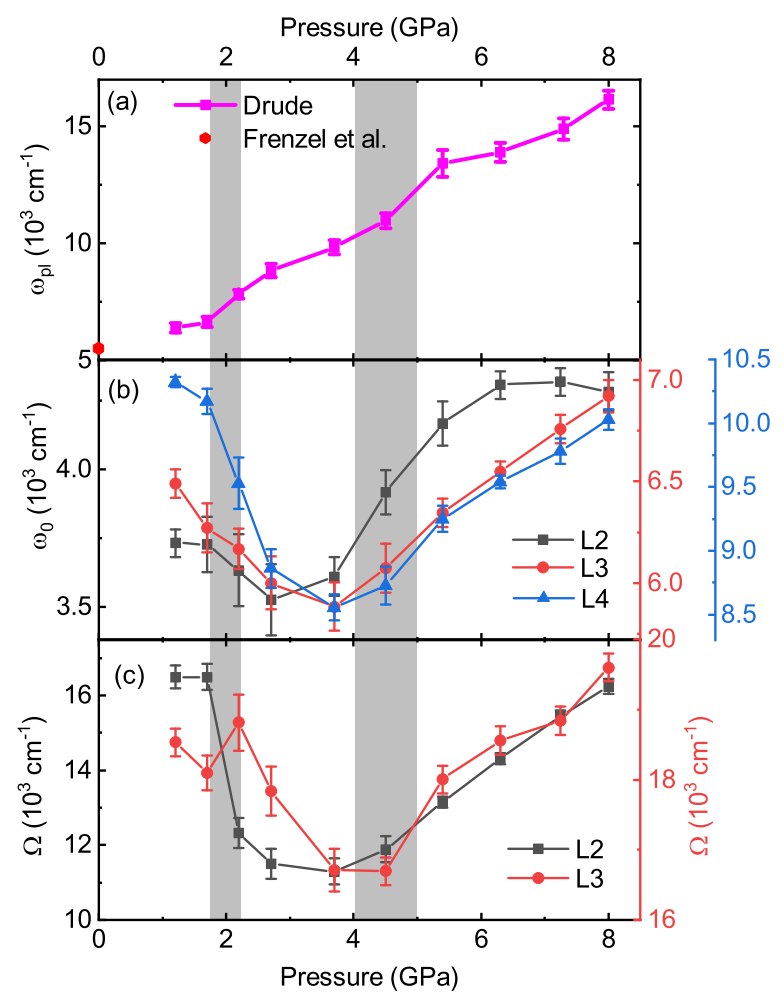

FIG. 4. (a) Plasma frequency of the Drude component, $\omega_{\mathrm{pl}, \mathrm{D}}$, as a function of pressure. The value of $\omega_{\mathrm{pl}, \mathrm{D}}$ at ambient pressure was extracted Frenzel et al. [37] as the average of the two measured polarization directions. (b) Resonance frequency $\omega_{0, j}$ of the Lorentz contributions L2 (black square), L3 (red dot), and L4 (blue triangle) as a function of pressure. (c) Oscillator strength, as given by $\Omega_{j}$ in Equ. (1), for the Lorentz contributions L2 (black square) and L3 (red dot) as a function of pressure. The vertical grey shaded areas mark the critical pressure ranges of the phase transitions as explained in the text.

ond anomaly at $\sim 4.5 \mathrm{GPa}$ in the pressure dependence of $\omega_{\mathrm{pl}, \mathrm{D}}$, namely a small step with a decrease of the slope of the curve.

Anomalies in the pressure dependence are also found for other fitting parameters. Around $2 \mathrm{GPa}$, the resonance frequency $\omega_{0}$ as well as the oscillator strength $\Omega_{j}$ of the low-energy Lorentz contributions L2 and L3 show a sudden drop and at $\sim 4.5 \mathrm{GPa}$ start to increase again as depicted in Fig. 4(b) and (c), respectively. Furthermore, at $\sim 2 \mathrm{GPa}$ the contribution L4 splits into two components, L4 and L5 (see grey arrows in Fig. 2). This splitting is responsible for the drop of the resonance frequency $\omega_{0}$ of L4 in Fig 4(b), and at $4.5 \mathrm{GPa}$ there is an additional inflection point, similar to L2 and L3. Besides, several Lorentz oscillators show anomalous behavior in their scattering rate at 2 and $4.5 \mathrm{GPa}$ (Fig. 6 in the appendix).

\section{DISCUSSION}

According to electronic band structure calculations for $\mathrm{T}_{\mathrm{d}}-\mathrm{WTe}_{2}$ under pressure by Pan et al. [16], we attribute the observed increasing metallicity stemming to the increasing size of the electron and hole pockets, and thus to an increasing density of states at the Fermi level $N\left(E_{\mathrm{F}}\right)$ upon pressure application. Lu et al. [30] confirmed this behavior by calculations for $1 \mathrm{~T}^{\prime}-\mathrm{WTe}_{2}$. The band structure and Fermi surface of the 1T' phase were stated to be very similar to the $T_{d}$ structure. Furthermore, a Lifshitz transition, i.e., a change in the shape of the Fermi surface [59], was found for pressures below $5 \mathrm{GPa}$ [30]. In fact, Cai et al. 20] experimentally observed a change of the Fermi surface topology already at $\approx 1 \mathrm{GPa}$. Since the shape of the Fermi surface directly influences the optical properties, we should see signatures of the Lifshitz transition in our optical data. Indeed, besides the onset of the increase of $\omega_{\mathrm{pl}, \mathrm{D}}$, the pronounced high-energy Lorentz contribution L4 splits into two components L4 and L5, of which the former shifts abruptly to lower energies between 1.7 and $2.2 \mathrm{GPa}$, whereas the latter shifts to higher energies upon further pressure increase. This manifests itself in the fact that we have to use slightly different fitting models to describe the spectra at low $(<$ $2 \mathrm{GPa}$ ) and at high (>2 GPa) pressures (see Fig. 2 and Fig. 5 and Fig. 6 in the appendix). Such a splitting of an absorption band is a strong indication for a phase transition, consistent with the observation of an electronic phase transition reported in Ref. [20]. Furthermore, our findings are consistent with the $a b$ initio calculations of Refs. [16, 30], where they observe that several electronic bands are pushed towards $E_{\mathrm{F}}$ and cross it between 0 and $5 \mathrm{GPa}$ without substantially changing the slope of the bands. Such changes in the bandstructure are further supported by an anomaly at $\sim 2 \mathrm{GPa}$ in the resonance frequency, oscillator strength, and scattering rate for several Lorentz contributions, as described above.

Furthermore, several Lorentz contributions show at $\sim 4.5 \mathrm{GPa}$ an inflection in their resonance frequency and oscillator strength, as well as a broadening. This inflection coincides with the aforementioned anomaly of $\omega_{\mathrm{pl}, \mathrm{D}}$ at around $4.5 \mathrm{GPa}$. We relate this second anomaly to the pressure-induced structural phase transition from $\mathrm{T}_{\mathrm{d}}$ - to $1 \mathrm{~T}$ '- $-\mathrm{WTe}_{2}$ reported in the literature: According to pressure-dependent XRD, Raman spectroscopy, and electrical transport measurements a $\mathrm{T}_{\mathrm{d}}$ to $1 \mathrm{~T}$ ' transition with a broad transition range from 6.0 to $15.5 \mathrm{GPa}$ occurs at room temperature, and superconductivity was observed in both polytypes 29] at low temperature. $\mathrm{Lu}$ et al. 30] identified a similar structural transition between $\sim 4$ and $11 \mathrm{GPa}$ at room temperature by XRD and Raman spectroscopy supported by ab initio calculations, proposing superconductivity to emerge from the high-pressure $1 \mathrm{~T}$ ' phase. Xia et al. 31] also found such a structural phase transition at $8-10 \mathrm{GPa}$ at room temperature in the plane-vertical Raman response, linking it to the emerging superconductivity [15] 60]. We note here that in the 
XRD study of Kang et al. [15] no signs for a structural phase transition were found under pressure, and therefore a Lifshitz transition at $10.5 \mathrm{GPa}$ was proposed to be responsible for the emerging superconductivity.

According to the similarity of the electronic band structures of $\mathrm{T}_{\mathrm{d}}$ - and $1 \mathrm{~T}^{\prime}-\mathrm{WTe}_{2}$, we don't expect to see a strong signature of the pressure-induced structural phase transition in the pressure-dependent optical conductivity. However, the anomalies in Fig. 4 are significant. Our data therefore support the occurrence of a phase transition at $\sim 4.5 \mathrm{GPa}$, coinciding with the reported structural phase transition from $\mathrm{T}_{\mathrm{d}^{-}}$to $1 \mathrm{~T}^{\prime}-\mathrm{WTe}_{2}$ [29 31]. As mentioned above, this phase transition is reported to occur across a rather broad pressure range.

\section{CONCLUSION}

According to the pressure dependence of the optical conductivity of $\mathrm{WTe}_{2}$, we observe a large transfer of spectral weight from high to low energies induced by external pressure. This finding points to strong changes in the electronic bandstructure of $\mathrm{WTe}_{2}$ under pressure. Furthermore, there are indications for two pressure-induced phase transitions at around 2 and $4.5 \mathrm{GPa}$ according to the pressure dependence of several optical parameters. The first transition most probably is of electronic type, i.e., it substantially affects the electronic bands, with a strong increase in the Drude plasma frequency. The transition at $\sim 4.5 \mathrm{GPa}$ corresponds to the structural phase transition from the $\mathrm{T}_{\mathrm{d}^{-}}$to the $1 \mathrm{~T}^{\prime}$ phase, where several optical parameters show an anomaly in their pressure evolution. Overall, our findings show the profound sensitivity of the bandstructure of $\mathrm{WTe}_{2}$ to external pressure.

\section{APPENDIX}

In the appendix we show additional optical data, namely the optical conductivity $\sigma_{1}(\omega)$, including the Drude-Lorentz fits and contributions, for all pressures (see Fig. 5), and the pressure evolution of all the parameters obtained from these fits (see Fig. 66).
[1] X.-C. Pan, X. Wang, F. Song, and B. Wang, "The study on quantum material $\mathrm{WTe}_{2}$," Advances in Physics: X 3, 1468279 (2018).

[2] W. Tian, W. Yu, X. Liu, Y. Wang, and J. Shi, "A review of the characteristics, synthesis, and thermodynamics of type-II Weyl semimetal WTe 2 ," Materials 11, 1185 (2018).

[3] P. K. Das, D. DiSante, C. Bigi, D. Kopic, A. Sterzi, J. A. Krieger, I. Vobornik, J. Fujii, T. Okuda, V. N. Strocov, M. B. H. Breese, F. Parmigiani, G. Rossi, S. Picozzi, R. Thomale, G. Sangiovanni, R. J. Cava, and G. Panaccione, "Electronic properties of candidate type-II Weyl semimetal $\mathrm{WTe}_{2}$. a review perspective," Electron. Struct. 1, 014003 (2019).

[4] A. A. Soluyanov, D. Gresch, Z. Wang, Q. S. Wu, M. Troyer, X. Dai, and B. A. Bernevig, "Type-II Weyl semimetals," Nature 527, 495 (2015).

[5] C.-L. Lin, R. Arafune, R.-Y. Liu, M. Yoshimura, B. Feng, K. Kawahara, Z. Ni, E. Minamitani, S. Watanabe, Y. Shi, M. Kawai, T.-C. Chiang, Iwao Matsuda, and N. Takagi, "Visualizing type-II Weyl points in tungsten ditelluride by quasiparticle interference." ACS Nano 11, 11459 (2017).

[6] P. Li, Y. Wen, X. He, Q. Zhang, C. Xia, Z.-M. Yu, S. A. Yang, Z. Zhu, H. N. Alshareef, and X.-X. Zhang, "Evidence for topological type-II Weyl semimetal WTe 2 ," Nature Commun. 8, 2150 (2017).

[7] M. N. Ali, J. Xiong, S. Flynn, J. Tao, Q. D. Gibson, L. M. Schoop, T. Liang, N. Haldolaarachchige, M. Hirschberger, N. P. Ong, and R. J. Cava, "Large, nonsaturating magnetoresistance in $\mathrm{WTe}_{2}$, , Nature 514, 205 (2014).

[8] Z. Zhu, X. Lin, J. Liu, B. Fauque, Q. Tao, C. Yang, Y. Shi, and K. Behnia, "Quantum oscillations, thermoelectric coefficients, and the fermi surface of semimetallic
WTe $2, "$ Phys. Rev. Lett. 113, 176601 (2015).

[9] S. Onishi, R. Jha, A. Miyake, R. Higashinaka, T. D. Matsuda, M. Tokunaga, and Y. Aoki, "Deviation from the kohler's rule and shubnikov-de haas oscillations in typeII Weyl semimetal $\mathrm{WTe}_{2}$ : High magnetic field study up to 56 T," AIP Advances 8, 101330 (2018).

[10] R. Jha, S. Onishi, R. Higashinaka, T. D. Matsuda, R. A. Ribeiro, and Y. Aoki, "Anisotropy in the electronic transport properties of Weyl semimetal $\mathrm{WTe}_{2}$ single crystals," AIP Advances 8, 101332 (2018).

[11] K. Kang, T. Li, E. Sohn, J. Shan, and K. F. Mak, "Nonlinear anomalous hall effect in few-layer WTe 2 ," Nature Materials 18, 324 (2019).

[12] O. O. Shvetsov, V. D. Esin, A. V. Timonina, N. N. Kolesnikov, and E. V. Deviatov, "Nonlinear hall effect in three-dimensional Weyl and Dirac semimetals," JETP Letters 109, 715 (2019).

[13] P. Sharma, F.-X. Xiang, D.-F. Shao, D. Zhang, E. Y. Tsymbal, A. R. Hamilton, and J. Seidel, "A roomtemperature ferroelectric semimetal," Sci. Adv. 5, 5080 (2019).

[14] K. G. Rana, F. K. Dejene, N. Kumar, C. R. Rajamathi, K. Sklarek, C. Felser, and S. S. P. Parkin, "Thermopower and unconventional nernst effect in the predicted type-II Weyl semimetal WTe 2 ," Nano Lett. 18, 6591 (2018).

[15] D. Kang, Y. Zhou, W. Yi, C. Yang, J. Guo, Y. Shi, S. Zhang, Z. Wang, C. Zhang, S. Jiang, A. Li, K. Yang, Q. Wu, G. Zhang, L. Sun, and Thao. Z., "Superconductivity emerging from a suppressed large magnetoresistant state in tungsten ditelluride," Nature Commun. 6, 7804 (2015).

[16] X.-C. Pan, X. Chen, H. Liu, Y. Feng, Z. Wei, Y. Zhou, Z. Chi, L. Pi, F. Song, X. Wan, Z. Yang, B. Wang, G. Wang, and Y. Zhang, "Pressure-driven dome-shaped superconductivity and electronic structural evolution in 



FIG. 5. Contributions to the pressure-dependent optical conductivity $\sigma_{1}$ of $\mathrm{WTe}_{2}$, as obtained from Drude-Lorentz fitting. The contribution L8 was kept constant for all fittings, as it is part of the high-energy extrapolation.

tungsten ditelluride," Nature Commun. 6, 7805 (2015).

[17] E. J. Sie, C. M. Nyby, C. D. Pemmaraju, S. J. Park, X. Shen, J. Yang, M. C. Hoffmann, B. K. Ofori-Okai, R. Li, A. H. Reid, S. Weathersby, E. Mannebach, N. Finney, D. Rhodes, D. Chenet, A. Antony, L. Balicas, J. Hone, T. P. Devereaux, T. F. Heinz, X. Wang, and A. M. Lindenberg, "An ultrafast symmetry switch in a Weyl semimetal," Nature 565, 61 (2019).
[18] H. Y. Lv, W. J. Lu, D. F. Shao, Y. Liu, S. G. Tan, and Y. P. Sun, "Perfect charge compensation in $\mathrm{WTe}_{2}$ for the extraordinary magnetoresistance: From bulk to monolayer," EPL 110, 37004 (2015).

[19] I. Pletikosic, M. N. Ali, A. V. Fedorov, R. J. Cava, and T. Valla, "Electronic structure basis for the extraordinary magnetoresistance in $\mathrm{WTe}_{2}$," Phys. Rev. Lett. 113, 216601 (2014). 

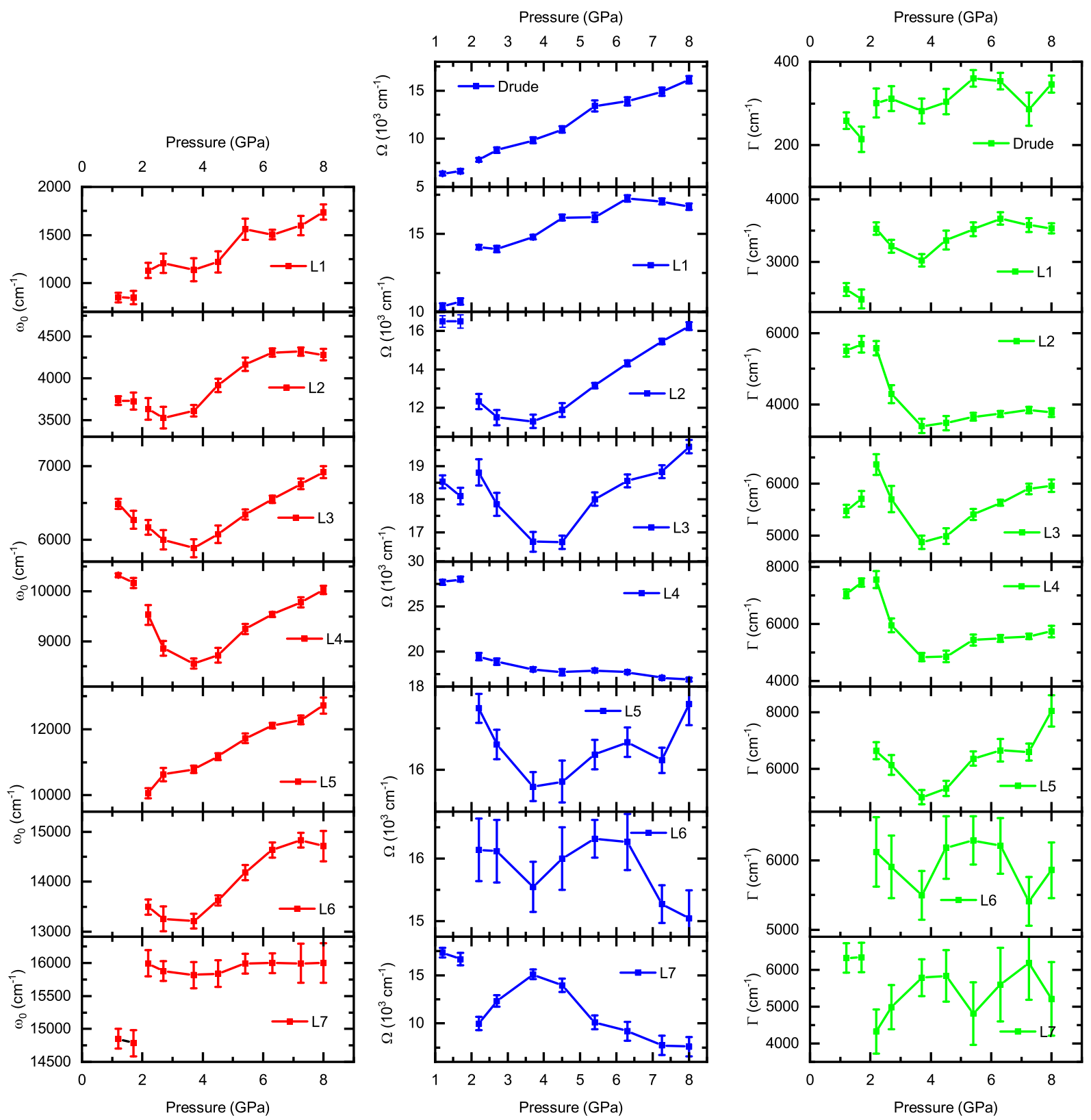

FIG. 6. Pressure-dependence of $\omega_{0}, \omega_{\mathrm{pl}, \mathrm{D}}, \Omega$, and $\Gamma$ of the various contributions of the Drude-Lorentz fittings shown in Fig. 5 and described in the main text.

[20] P. L. Cai, L. P. He, J. Pan, X. C. Hong, Z. Zhang, J. Zhang, J. Wei, Q. Mao, and S. Y. Li, "Drastic pressure effect on the extremely large magnetoresistance in $\mathrm{WTe}_{2}$ : Quantum oscillation study," Phys. Rev. Lett. 115, 057202 (2015).

[21] X.-C. Pan, Y. Pan, J. Jiang, H. Zuo, H. Liu, X. Chen, Z. Wei, S. Zhang, Z. Wang, X. Wan, Z. Yang, D. Feng, Z. Xia, L. Li, F. Song, B. Wang, Y. Zhang, and G. Wang, "Carrier balance and linear magnetoresistance in type-II Weyl semimetal WTe 2 ," Front. Phys. 12, 127203 (2017).

[22] Y. Wang, L. Wang, X. Liu, H. Wu, P. Wang, D. Yan, B. Cheng, Y. Shi, K. Watanabe, T. Taniguchi, S.J. Liang, and F. Miao, "Direct evidence for charge compensation-induced large magnetoresistance in thin $\mathrm{WTe}_{2}, "$ Nano Lett. 19, 3969 (2019).

[23] Y. Luo, H. Li, Y. M. Dai, H. Miao, Y. G. Shi, H. Ding, A. J. Taylor, D. A. Yarotski, R. P. Prasankumar, and J. D. Thompson, "Hall effect in the extremely large magnetoresistance semimetal WTe $\mathrm{W}_{2}$," Appl. Phys. Lett. 107, 182411 (2015).

[24] Y. Wang, K. Wang, J. Reutt-Robey, J. Paglione, and M. S. Fuhrer, "Breakdown of compensation and persistence of nonsaturating magnetoresistance in gated $\mathrm{WTe}_{2}$ thin flakes," Phys. Rev. B 93, 121108 (2016).

[25] D. Rhodes, S. Das, Q. R. Zhang, B. Zeng, N. R. Pradhan, N. Kikugawa, E. Manousakis, and L. Balicas, "Role of 
spin-orbit coupling and evolution of the electronic structure of $\mathrm{WTe}_{2}$ under an external magnetic field," Phys. Rev. B 92, 125152 (2015).

[26] J. Jiang, F. Tang, X. C. Pan, H. M. Liu, X. H. Niu, Y. X. Wang, D. F. Xu, H. F. Yang, B. P. Xie, F. Q. Song, P. Dudin, T. K. Kim, M. Hoesch, P. K. Das, I. Vobornik, X. G. Wan, and D. L. Feng, "Signature of strong spinorbital coupling in the large nonsaturating magnetoresistance material $\mathrm{WTe}_{2}$," Phys. Rev. Lett. 115, 166601 (2015).

[27] L. R. Thoutam, Y. L. Wang, Z. L. Xiao, S. Das, A. Lucian-Mayer, R. Divan, G. W. Crabtree, and W. K. Kwok, "Temperature-dependent three-dimensional anisotropy of the magnetoresistance in WTe 2 ," Phys. Rev. Lett. 115, 046602 (2015).

[28] P. He, C.-H. Hsu, S. Shi, K. Cai, J. Wang, Q. Wang, G. Eda, H. Lin, V. M. Peireira, and H. Yang, "Nonlinear magnetotransport shaped by fermi surface topology and convexity," Nature Commun. 10, 1290 (2019).

[29] Y. Zhou, X. Chen, N. Li, R. Zhang, X. Wang, C. An, Y. Zhou, X. Pan, F. Song, B. Wang, W. Yang, Z. Yang, and Y. Zhang, "Pressure-induced $\mathrm{T}_{d}$ to $1 \mathrm{~T}$ ' structural phase transition in $\mathrm{WTe}_{2}, "$ AIP Advances 6, 075008 (2016).

[30] P. Lu, J.-S. Kim, J. Yang, H. Gao, J. Wu, D. Shao, B. Li, D. Zhou, J. Sun, D. Akinwand, D. Xing, and J.-F. Lin, "Origin of superconductivity in the Weyl semimetal $\mathrm{WTe}_{2}$ under pressure," Phys. Rev. B 94, 224512 (2016).

[31] J. Xia, D.-F. Li, J.-D. Zhou, P. Yu, J.-H. Lin, J.-L. Kuo, H.-B. Li, Z. Liu, J.-X. Yan, and Z.-X. Shen, "Pressureinduced phase transition in Weyl semimetallic WTe, Small 13, 1701887 (2017).

[32] Y. T. Chan, P. L. Alireza, K. Y. Yip, Q. Niu, K. T. Lai, and S. K. Goh, "Nearly isotropic superconductivity in the layered Weyl semimetal WTe 2 at 98.5 kbar," Phys. Rev. B 96, 180504(R) (2017).

[33] C. J. Tabert, J. P. Carbotte, and E. J. Nicol, "Optical and transport properties in three-dimensional Dirac and Weyl semimetals," Phys. Rev. B 93, 085426 (2016).

[34] B. Xu, Y. M. Dai, L. X. Zhao, K. Wang, R. Yang, W. Zhang, J. Y. Liu, H. Xiao, G. F. Chen, A. J. Taylor, D. A. Yarotski, R. P. Prasankumar, and X. G. Qiu, "Optical spectroscopy of the Weyl semimetal TaAs," Phys. Rev. B 93, 121110(R) (2016).

[35] J. P. Carbotte, "Dirac cone tilt on interband optical background of type-I and type-II Weyl semimetals," Phys. Rev. B 94, 165111 (2016).

[36] C. C. Homes, M. N. Ali, and R. J. Cava, "Optical properties of the perfectly compensated semimetal WTe $\mathrm{W}_{2}$," Phys. Rev. B 92, 161109(R) (2015).

[37] A. Frenzel, C. C. Homes, Q. D. Gibson, Y. M. Shao, K. W. Post, A. Charnukha, R. J. Cava, and D. N. Basov, "Anisotropic electrodynamics of type-II Weyl semimetal candidate WTe 2 ," Phys. Rev. B 95, 245140 (2017).

[38] S.-I. Kimura, Y. Nakajima, Z. Mita, Rajveer Jha, R. Higashinaka, T. D. Matsuda, and Y. Aoki, "Optical evidence of the type-II Weyl semimetals $\mathrm{MoTe}_{2}$ and $\mathrm{WTe}_{2}$," Phys. Rev. B 99, 195203 (2019).

[39] M. Chinotti et al., Phys. Rev. B 94, 245101 (2016).

[40] D. Chaudhuri, B. CHeng, A. Yaresko, Q. D. Gibson, R. J. Cava, and N. P. Armitage, Phys. Rev. B 96, 075151 (2017).

[41] D. Rodriguez, A. A. Tsirlin, T. Biesner, T. Ueno, T. Takahashi, K. Kobayashi, M. Dressel, and E. Uykur,
"Two linear regimes in optical conductivity of a type-I Weyl semimetal: The case of elemental tellurium," Phys. Rev. Lett. 124, 136402 (2020).

[42] F. LeMardele, D. Santos-Cottin, E. Martino, S. Semeniuk, S. B. David, F. Orbanic, M. Novak, Z. Rukelj, C. C. Homes, and A. Akrap, "Optical conductivity of the typeII Weyl semimetal TaIrTe $e_{4}, "$ arXiv:2004.00147v1.

[43] B. E. Brown, "The crystal structures of $\mathrm{WTe}_{2}$ and hightemperature $\mathrm{MoTe}_{2}$," Acta Cystallogr. 20, 268 (1966).

[44] W. G. Dawson and D. W. Bullet, "Electronic structure and crystallography of $\mathrm{MoTe}_{2}$ and $\mathrm{WTe}_{2}, "$ J. Phys. C: Solid State Phys. 20, 6159 (1987).

[45] H. K. Mao, J. Xu, and P. M. Bell, "Calibration of the ruby pressure gauge to $800 \mathrm{kbar}$ under quasi-hydrostatic conditions," J. Geophys. Res. 91, 4673 (1986).

[46] K. Syassen, "Ruby under pressure," High Pressure Res. 28, 75 (2008).

[47] A. Pashkin, M. Dressel, and C. A. Kuntscher, "Pressureinduced deconfinement of the charge transport in the quasi-one-dimensional Mott insulator (TMTTF) ${ }_{2} \mathrm{AsF}_{6}$," Phys. Rev. B 74, 165118 (2006).

[48] D. B. Tanner, "Use of x-ray scattering functions in Kramers-Kronig analysis of reflectance," Phys. Rev. B 91, 035123 (2015).

[49] M. K. Jana, A. Singh, D. J. Late, C. R. Rajamathi, K. Biswas, C. Felser, U. V. Waghmare, and C. N. R. Rao, "A combined experimental and theoretical study of the structural, electronic and vibrational properties of bulk and few-layer Td-WTe 2 ," J. Phys.: Condens. Matter 27, 285401 (2015).

[50] P. E. C. Ashby and J. P. Carbotte, "Chiral anomaly and optical absorption in Weyl semimetals," Phys. Rev. B 89, 245121 (2014).

[51] We point out that the features in our optical conductivity spectra are rather broad compared to literature data, since the measurements were carried out under external pressure at room temperature.

[52] The small scattering rate and its temperature dependence as well as the rapid increase of the effective electron number of the second Drude term are concluded to be due to Weyl-type carriers 38].

[53] D. Santos-Cottin, E. Martino, F. LeMardele, C. Witteveen, F. O. vonRohr, C. C. Homes, Z. Rukelj, and A. Akrap, "Low-energy excitations in type-II Weyl semimetal $\mathrm{T}_{d}-\mathrm{MoTe}_{2}$ evidenced through optical conductivity," Physical Review Materials 4, 021201(R).

[54] D. Vollhardt, "Characteristic crossing points in specific heat curves of correlated systems," Phys. Rev. Lett. 78, 1307 (1997).

[55] M. Greger, M. Kollar, and D. Vollhardt, "Isosbestic points: How a narrow crossing region of curves determines their leading parameter dependence," Phys. Rev. B 87, 195140 (2013).

[56] S. Uchida, T. Ido, H. Takagi, T. Arima, Y. Tokura, and S. Tajima, "Optical spectra of $\mathrm{La}_{2-x} \mathrm{Sr}_{x} \mathrm{CuO}_{4}$ : Effect of carrier doping on the electronic structure of the $\mathrm{CuO}_{2}$ plane," Phys. Rev. B 43, 7942 (1991).

[57] I. Kezsmarki, N. Hanasaki, D. Hashimoto, S. Iguchi, Y. Taguchi, S. Miyasaka, and Y. Tokura, "Charge dynamics near the electron-correlation induced metalinsulator transition in pyrochlore-type molybdates," Phys. Rev. Lett. 93, 266401 (2004).

[58] M. Dressel and G. Grüner, Electrodynamics of Solids: Optical Properties of Electrons in Matter (Cambridge 
University Press, 2002).

[59] I. M. Lifshitz, "Anomalies of electron characteristics of a metal in the high pressure region," Sov. Phys. JETP 11, 1130 (1960).

[60] One has to note the different pressures at which superconductivity has been observed, namely 2.5 (no PTM) [16], 4.0 (PTM: Daphne 7373) [29] and 10.5 GPa (PTM: $\mathrm{NaCl}$ ) 15], as well as the different pressure ranges of the structural phase transition, namely 6-15.5 (PTM: Daphne 7373) [29], 4-11 (PTM: Ar, Ne) [30] and 8-
10 GPa (PTM: methanol-ethanol 4:1 mixture) 31]. The discrepancies have not been clarified but might be related to the different pressure transmitting media used. Additionally, the crystal quality might have an influence on the electronic properties of $\mathrm{WTe}_{2}$ [61].

[61] M. N. Ali, L. Schoop, J. Xiong, S. Flynn, Q. Gibson, M. Hirschberger, N. P. Ong, and R. J. Cava, "Correlation of crystal quality and extreme magnetoresistance of $\mathrm{WTe}_{2}, " \mathrm{EPL}$ 110, 67002 (2015). 